\title{
DIO: Development of a Mobile Application for Effective Information Sharing and Collaboration among Co-Located People
}

\author{
Jun-Seok Kwak ${ }^{1}$, Jae-hwan Jin ${ }^{1}$ and Myung-Joon Lee ${ }^{2 *}$ \\ Department of Electrical/Electronic and Computer Engineering, University of \\ Ulsan, 93, Daehak-ro, Nam-gu, Ulsan 680-749, Republic of Korea \\ ${ }^{1}$ \{calintsz,jjhok2000\}@naver.com, ${ }^{2 *}$ mjlee@ulsan.ac.kr
}

\begin{abstract}
Since social activities of the modern people have increased continuously in recent year, many people frequently communicate with each other online and/or offline, exchanging a variety of information. Thanks to the development of smartphones and a wide range of mobile applications helping social activities of people, people can communicate more easily beyond their time and space. But, there are not many mobile applications specially designed for effective communication and collaboration among co-located people, most of the existing applications only provide limited functions. In this paper, we present an android mobile application DIO specially designed for co-located people to collaborate in a rich way. DIO enables users to share and exchange their profiles, favorite photos, musics, videos and documents according to one of the three open levels - public, business and community level. In addition, through DIO, co-located people can chat, vote, and survey. DIO is implemented on the top of AllJoyn platform - an open source IoT platform developed by the AllSeen Alliance. So, it can be easily done to extend DIO to communicate with other smart things running on the same platform.
\end{abstract}

Keywords: Android, Smartphone, AllJoyn, DIO, Information Sharing, Collaboration

\section{Introduction}

As a variety of social network services have proliferated over the last decade, social activities of people have rapidly increased. Modern people need to talk with others, get an answer from them and perform survey of these activities, but overhead to communicate with each other is also becoming bigger. Thus, getting over the limitations of time and space, effective communication methods would be helpful to reduce the overhead, sharing information promptly and easily. Smart devices that most of the modern people are using as the communication means support a variety of communication protocols such as Bluetooth, RFID, Wi-Fi and Wi-Fi Direct. Based on these communication methods, there are applications with information sharing among co-located people such as CardShake [1], business card holder with NFC[2], Phriz.be[3], and EXO U [4-5]. However, these applications only support limited functionality such as file transfer, sharing Information, educational collaboration, business card exchange.

In this paper, we present an android mobile application DIO (Do It Ourselves) specially designed for co-located people to collaborate in a rich way. For this end, DIO provides useful features for sharing their personal information, enabling them to share and exchange their profiles, favorite photos, musics, videos and documents according to one of the three open levels - public, business and community level. According to the characteristics of the meeting that a DIO user participates in, the user can set beforehand the open level to the personal information. At off-line social meetings, people usually chat with nearby participants and vote for making

${ }^{*}$ Corresponding Author 
decisions. Often, they also perform a survey to find out the tendency of their minds or activities. DIO also supports these activities as main collaboration features. DIO is implemented on the top of the AllJoyn [8] platform. AllJoyn is an open source IoT platform developed by the AllSeen Alliance [9] to make various IoT applications/devices to work together regardless of the brand, OS, transport layer. Since DIO developed as one of AllJoyn applications, it can be easily extended for communicating with AllJoyn IoT applications/devices, or can be naturally incorporated with other AllJoyn applications.

This paper is the extension of our previous work [10], being organized as follows. In Section 2, we introduce the background knowledge of AllJoyn Framework and some related applications. In Sections 3, we describe the system structure and collaboration features of the DIO application, while presenting the user interface and the associated functionalities of DIO. In Section 5, the features of the DIO are compared with some related applications. We conclude the paper in Section 6.

\section{Background Knowledge}

In this section, we describe the AllJoyn framework and some related mobile applications for co-located people.

\subsection{AllJoyn Framework}

The AllSeen Alliance is managing the development of the AllJoyn as an open source framework. The AllJoyn framework and core system services let compatible devices and applications find, communicate and collaborate with each other beyond the boundaries of product category, platform, brand, and connection type. The most important feature in AllJoyn is the AllJoyn software bus that puts all things together. The AllJoyn software bus is extended on the basis of the D-Bus [11] protocol. An AllJoyn bus is formed through the connection of the AllJoyn routers running as background processes. AllJoyn applications get connected to the bus by connecting to the router through the Bus Attachment object. Each Bus Attachment is assigned a unique name, containing the addresses of its service objects in a tree format. Each service object should implement the AllJoyn bus interface.

\subsection{Related Mobile Applications}

Phriz.be is a file exchange application using the AllJoyn platform, enabling co-located users to exchange files such as photos, contacts and videos through smart phones, tablet PCs, so on. EXO $U$ is also an AllJoyn application which allows co-located users to collaborate for education purpose. It supports simple quiz, sharing of text information and pdf files, and screen control functions. CardShake is a business card exchange application, allowing users to exchange business cards through Bluetooth or NFC. With WhoAmI, any type of file and business card information can be exchanged through Wi-Fi or Wi-Fi Direct. However, these applications are not enough to be used as an effective collaboration tool because they only provide a few limited collaboration functions.

\section{Structure and Design of DIO}

In this section, we describe the system structure and collaboration features of DIO.

\subsection{The System Structure of DIO}

Since DIO is an AllJoyn application, DIO runs along with an AllJoyn router. In the system structure, View Logic is a region that implements the DIO user interface. DIO Application Context Object is a shared object that contains the state and information of 
DIO. Network IO Module manages the network input and output, while FileIO manages the input and output of the local file. TextPref Module manages the text configuration file, and AllJoyn Manager manages session initialization, generation, and connection. The overall structure of DIO application is as shown in Figure 1.

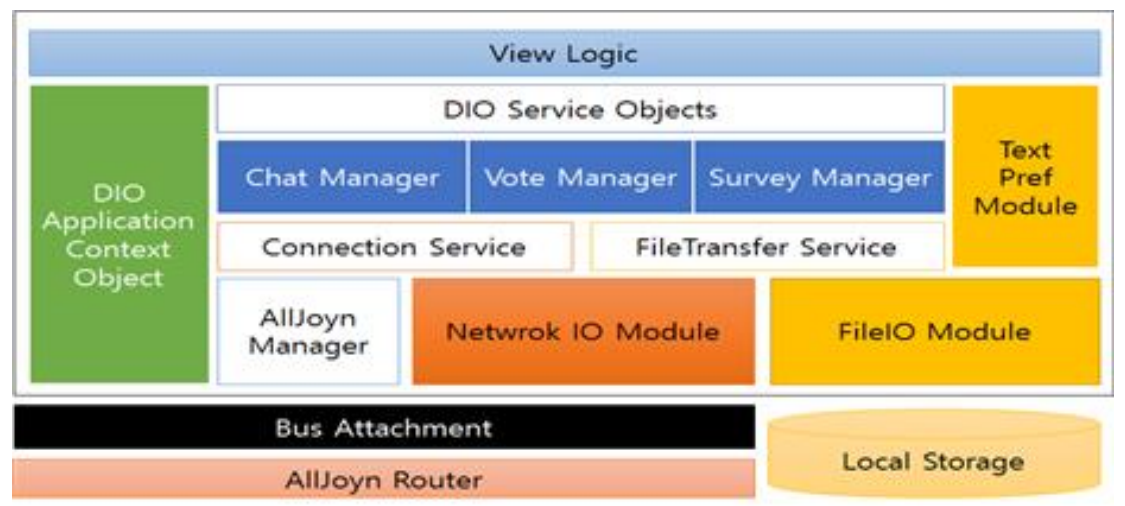

Figure 1. DIO System Structure

A DIO application running on an android device provides bidirectional services to the DIO applications running on other devices. Chat Manger, Vote Manager, and Survey Manger are different kinds of Service Manager, creating different DIO service objects such as chat room, vote room and survey room. In addition, Service Managers manages a list of service objects, updating the list when a new service object is created. The relationship between Service Manager and service objects is shown in Figure 2. They communicate with each other via AllJoyn bus.

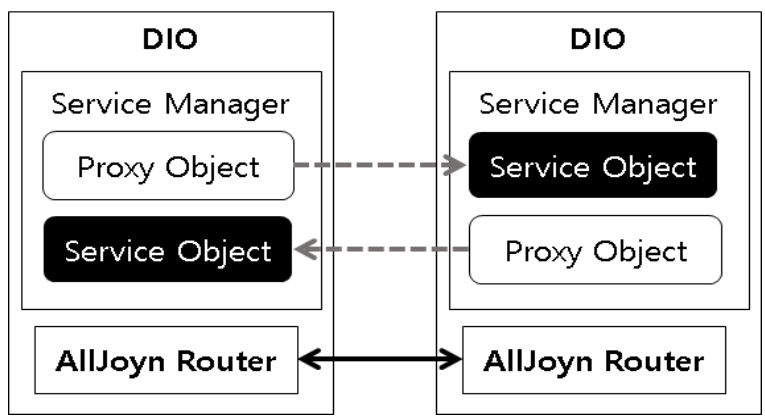

\section{Figure 2. Relationship between Service Manager and Service Objects}

Each object for chat, votes, or surveys room is registered on the bus as a service object. Performing the invoked method, it provides the associated service. To communicate with each other, DIO application first gets the proxy object from the bus, using this proxy object to call a remote method. Connection Service manages the information of the connected users, and FileTransfer Service is responsible for the file transmission and reception.

FileTransfer Service is a thread running background, transmitting files when other users requests resources. The information of the process transferring and copying remote resources is stored in Work object. FileTransfer Service manages the list of such Work objects. Connection Service is also a thread running background that manages a list of user information by requesting and saving the information of the users when new users are added to the session. 


\subsection{Initialization of DIO Session}

As soon as connecting to the AllJoyn router, to discover the DIO service, DIO registers the DIO application service information and callback listeners to the router and searches the service.

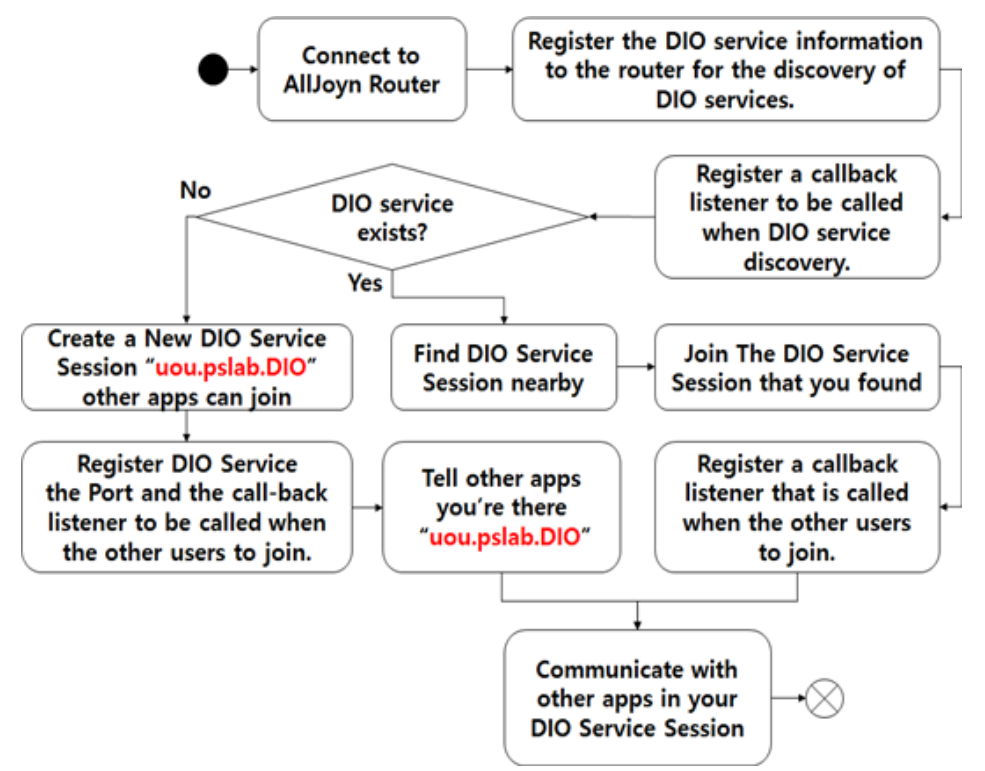

Figure 3. Initialization Process of DIO Application Session

If the service session already exists, DIO accesses to the service session. To add other users, it registers the callback listeners and communicates with other users by saving the information of other users through the callback method. If the service does not exist, it creates a new session and registers a callback listener which will be summoned when other users participate. Also, it advertises "uou.pslab.DIO" as the well-known name of DIO application. It saves the information on other users through the callback method to communicate with other users. This initialization process of the DIO session is described in Figure 3 as an activity diagram.

\subsection{Information Sharing Features of DIO}

The user can set the share level of the resources. Share level consists of three levels: public, business and community level. The public level is a level for the personal information open to all users, and the business level is a level for the personal information open to a business group, and the community level is a level for the personal information only to the community group. Only the users in the share level which is equal or higher than share level of the resource can access the resource. Using DIO, co-located users can exchange business card information or resource files such as musics, photos, videos, documents in a controlled way. Users can create different business cards according to the share level. Only the card information lower than or equal to the current application share level is open to other users.

\subsection{DIO Collaboration Features}

As mentioned earlier, DIO provides collaboration features for co-located people such as chatting with nearby participants, voting for making decisions, and surveying to find out the tendency of their activities. To broadcast a chat message, it gets first the list of the connected users. Then, as shown in Figure 4, it calls the broadcastChattingRoom method of each user which is implemented for message deliver. 


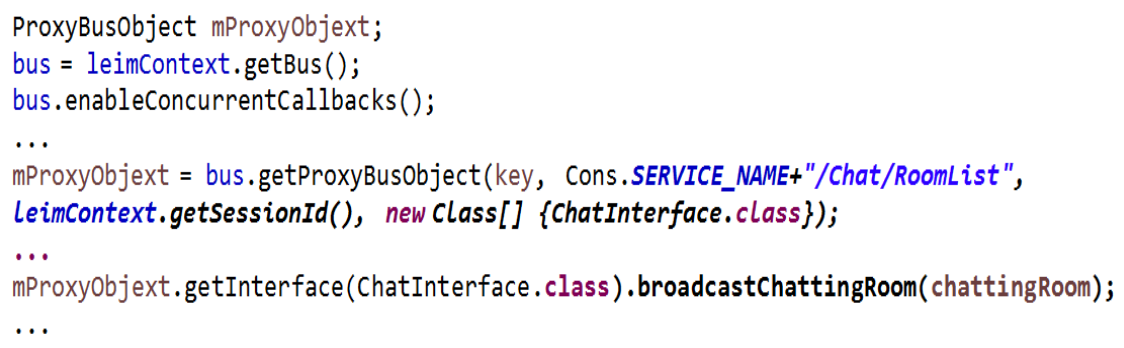

Figure 4. Method Invocation for BroadcastChattingRoom

The detailed process of conducting or participating in a survey is shown in Figure 5 as an activity diagram. The user can create a survey room, can participate in a survey, can request the result of a survey, and can store the result in the local storage. To perform a survey, the survey room information is broadcasted after writing out the questions for a new survey room.

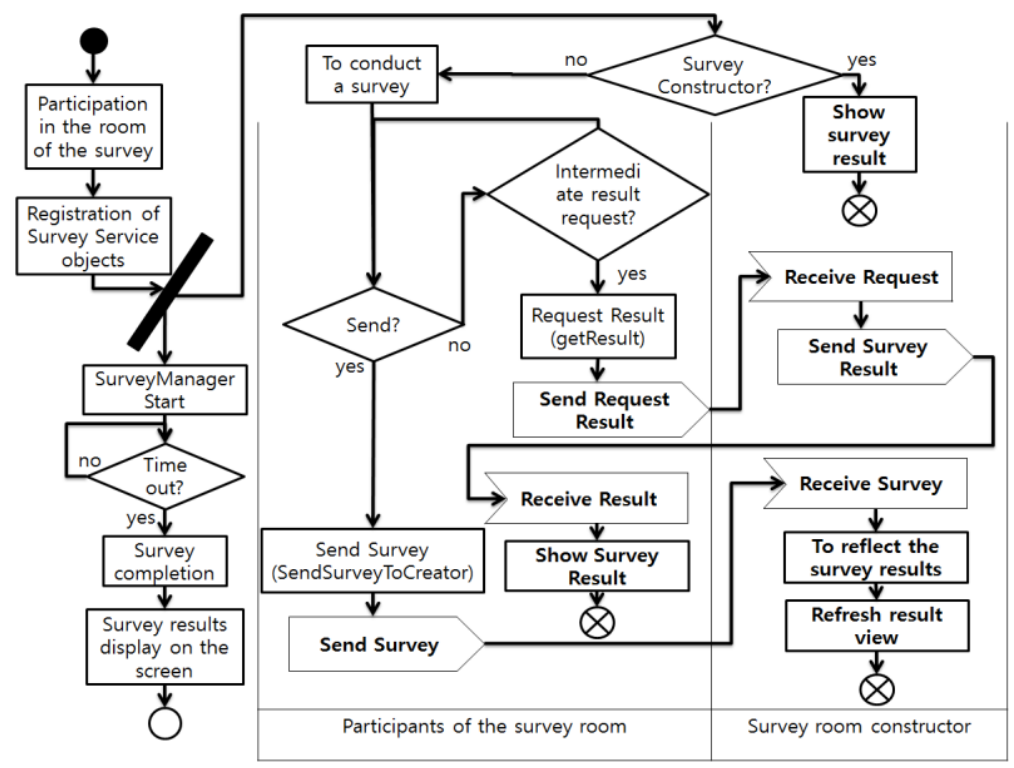

Figure 5. The Survey Activity Diagram

When a user participates in a survey room, it first registers the service object to provide a survey service. Then, it runs a thread which checks the end time of the survey to know when to close the survey. While the screen displays the results of surveys to the survey creator, it displays the questions of the survey to the participants. If a participant presses the Send button to complete the survey, the survey content of the participant is sent to the survey creator, reflected to the intermediate result of the survey. If the participant presses the button to request the intermediate result, the creator sends the object that contains the intermediate survey result. The received intermediate result can be saved and viewed again at any time. The process of voting is similar to the survey process.

\section{Interface and Operation of DIO}

In this section, we present the user interface of DIO in accordance with the collaborative functionalities of DIO. 


\subsection{The Main Screen and Information Sharing Screen of DIO}

The main screen of the DIO is composed of four tabs: user list, chat list, vote list, and survey list. Figure 6 shows the main screen, the user profile screen and the resource sharing screen of DIO. A user can change the detailed information at any time through the edit profile button in the main screen. Information sharing is divided into the business card information exchange and the resources exchange.
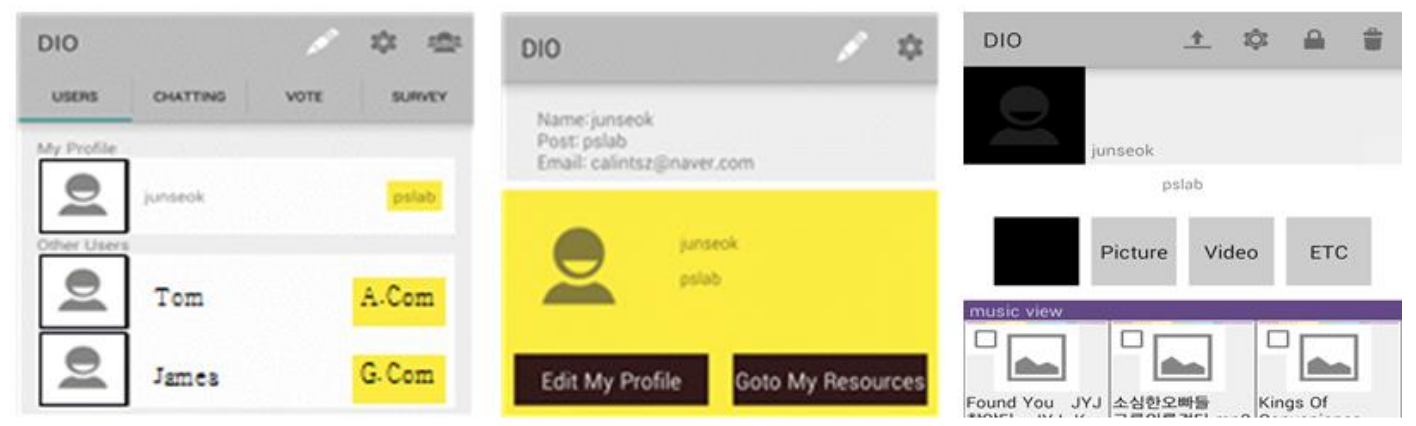

Figure 6. Main Screen, User Profile Screen and Resource Sharing Screen

4.1.1. The Screen of the Business Card Information Exchange: When a user launches DIO, it first requests the basic user information stored as a key-value pairs in a text file. The Copy button is displayed at the lower left corner of the screen of business card information of other users. When one of the other users presses the copy button, the card information file is transferred to the user who pressed the button.

4.1.2. Resource Sharing Screen: As we can see at the screen of shared resources in Figure 6, through the register resource button at the top of the screen, a user can press and select a resource. By the Confirm button, the dialog window to select the share folder for the resource appears. When you select a share folder, then the dialog box with the share level of the resource comes up. By setting the share level of the resource, the resource is registered. When you select a shared level, it is stored in the text configuration file as a key-value pair whose value is the shared level and key is the address of the resource.

\subsection{Collaboration Screen of DIO}

4.2.1. Chat Screen: The list of chat rooms appears on this screen. Through the room creating button at the bottom right of the screen, a user can create a new chat room with the room name. Then, the room information is broadcasted to the connected users.

4.2.2. Vote Screen: A vote room is created by the create vote room button at bottom right of the screen. The screen of the create vote room is shown in Figure 7. If the user creating the vote room fills out the polls and presses the Confirm button, then the vote room information is broadcasted to the connected users.

The screens to perform a vote are also shown in Figure 7. Through the Vote button, the result of the vote is transferred to the user who created the vote room. The intermediate result of the vote can be requested as in the case of survey. If the user who created the vote room can stop the vote through the stop button and can delete the vote room by the Delete button. Through the save button, the result of the vote is saved and can be viewed again at any time. 

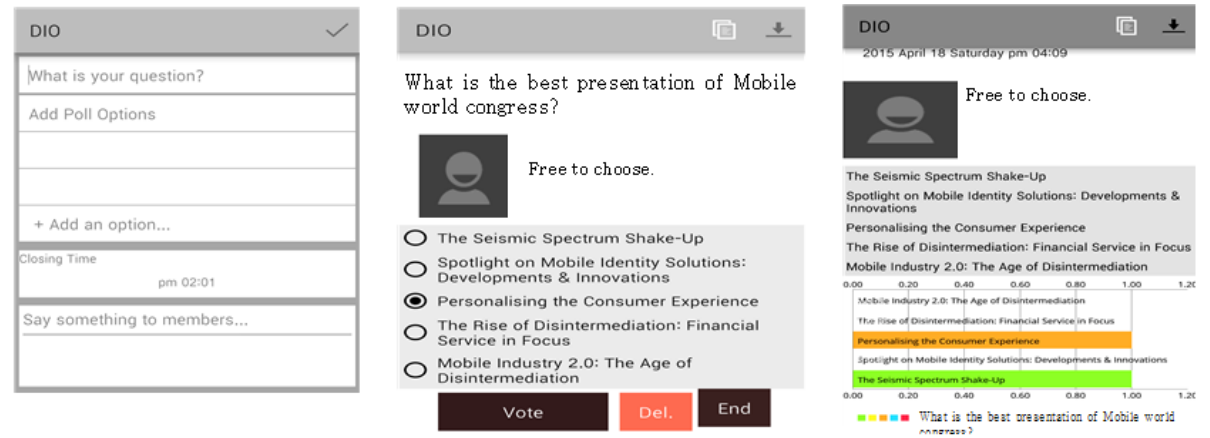

Figure 7. Setting Screen, Vote and Result Screen of DIO

4.2.3. Survey Screen: Figure 8 shows the screens of a survey room. The screen of a survey displays multiple-choice and short-answer questions. Participants can send answers to the survey creator by the Confirm button at the bottom of the screen. The survey creator can see the result and can also save the result through the save button at the top of screen. The survey participants can request the intermediate survey result by the request button at the top of the screen. Likewise, participants can save the survey result to show the result again at any time.
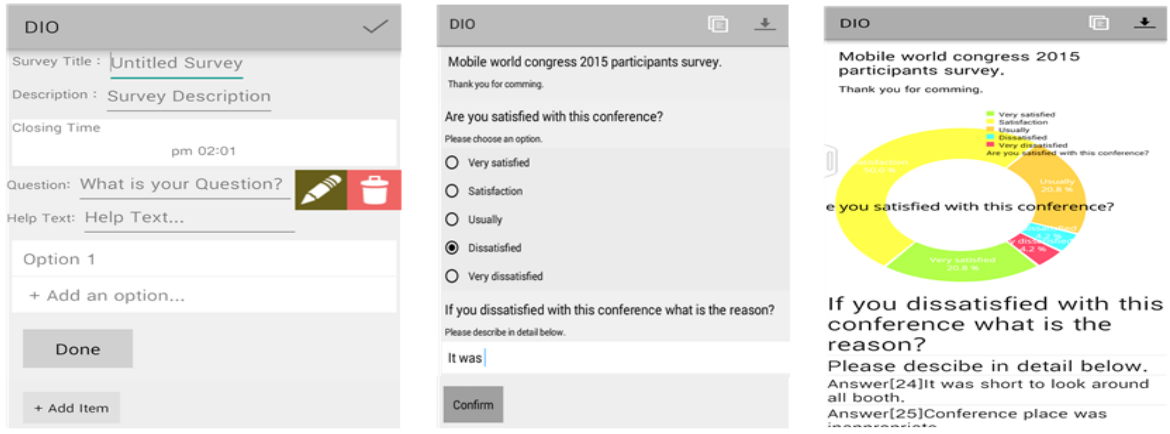

Figure 8. Survey Creating Screen, Survey and Result Screen

\section{Feature Comparison and Evaluation of DIO}

Phriz.be is an AllJoyn application which supports transferring photos, videos, contacts. WhoAmI enables co-located users to exchange files and business card information over the Wi-Fi and Wi-Fi direct environment. EXO $\mathrm{U}$ is also an AllJoyn application which supports collaboration between co-located people for educational purposes. EXO U provides simple multiple-choice and short-answer quiz for collaboration. However, the type of file that can be exchanged is limited, so it is inconvenient to exchange various types of information without overhead. CardShake is a business card information exchange application for nearby people. Table 1 shows the detailed feature comparison of DIO and the related applications, clarifying the rich collaboration features of DIO in addition to the effective resource sharing mechanism. 
Table 1. Comparison Table of Co-location a Short Distance of Information Exchange and Collaboration Applications

\begin{tabular}{|c|c|c|c|c|c|}
\hline $\begin{array}{l}\text { Application } \\
\text { Items }\end{array}$ & Phriz.be & EXO U & CardShake & WhoAmI & DIO \\
\hline Operating system & $\begin{array}{c}\text { Windows 8.1, } \\
\text { Windows } \\
\text { Phone 8.1 }\end{array}$ & $\begin{array}{l}\text { Android, } \\
\text { iOS, } \\
\text { Mac OS, } \\
\text { Windows }\end{array}$ & $\begin{array}{l}\text { Android, } \\
\text { iOS }\end{array}$ & Android & Android \\
\hline $\begin{array}{c}\text { Communication } \\
\text { technology }\end{array}$ & AllJoyn & AllJoyn & $\begin{array}{c}\text { NFC, } \\
\text { Blutooth }\end{array}$ & $\begin{array}{c}\text { Wi-Fi, } \\
\text { Wi-Fi Direct }\end{array}$ & AllJoyn \\
\hline $\begin{array}{l}\text { Resource } \\
\text { exchange }\end{array}$ & $\checkmark$ & - & $\triangle$ & $\checkmark$ & $\checkmark$ \\
\hline $\begin{array}{l}\text { Resources } \\
\text { Type }\end{array}$ & $\begin{array}{c}\text { Photos, } \\
\text { Contact, } \\
\text { Video }\end{array}$ & - & $\begin{array}{c}\text { Business } \\
\text { card } \\
\text { information }\end{array}$ & All types & All types \\
\hline $\begin{array}{l}\text { Collaboration } \\
\text { features }\end{array}$ & - & $\begin{array}{c}\text { Quiz, Class, } \\
\text { Screen control }\end{array}$ & - & - & $\begin{array}{l}\text { Chat, } \\
\text { Vote, } \\
\text { Survey }\end{array}$ \\
\hline
\end{tabular}

( - : unsupported, $\triangle$ : partially supported, $\checkmark$ : supported )

\section{Conclusion}

DIO is a specially designed mobile application for co-located people to collaborate in a versatile way. Through DIO, users can spontaneously exchange their personal information according to the one of three open levels: public, business and community level. In association with the characteristics of the meetings they are participating in, users can designate the open levels, introducing themselves without overhead. This feature enables users to share each other's favorite musics, photos, videos and others, helping to understand each other more widely and deeply. Moreover, DIO provides useful collaboration features for co-located people such as chat, vote and survey, which are usually performed at a variety of off-line meetings. Thanks to these novel features, DIO would be of good use for various circumstances including seminars, conferences, and consumer meetings. AllJoyn, over which DIO is implemented, is considered as one of several emerging platforms to make various IoT applications/devices to work together. This ensures the extensibility of DIO in communicating with other IoT applications/devices for higher level of collaboration.

\section{Acknowledgments}

This research was partially supported by Basic Science Research Program through the National Research Foundation of Korea (NRF) funded by the Ministry of Education (No. 2013R1A1A4A01004459).

\section{References}

[1] CardShake, https://play.google.com/store/apps/details?id=Tesla.Android.CardShake\&hl=en

[2] Business Card Holder with NFC,

https://play.google.com/store/apps/details?id=kr.co.atsolution.android.nbc\&hl=en 
[3] Phriz.be, https://developer.qualcomm.com/showcase/phrizbeproximity-based-mobile-sharing-lplatform

[4] EXO U, http://www.exou.com/

[5] H. Falaki, R. Mahajan, S. Kandula, D. Lymberopoulos, R. Govindan and D. Estrin, "Diversity in smartphone usage", In Proceedings of the 8th international conference on Mobile systems, applications, and services, June (2010).

[6] J. S. Kwak, J. M. Park and M. J. Lee, "WhoAmI: Personal Information Sharing Application over WiFi and WiFi Direct", J. Korea Inst. Inf. Commun. Eng., vol. 18, no. 2, (2014), pp. 371-378.

[7] Burnette E., "Hello, Android: introducing Google's mobile development platform", Pragmatic Bookshelf, (2009).

[8] AllJoyn, "Introduction to the AllJoyn framework", https://allseenalliance.org/docsanddownloads/documentation/introduction-alljoynframework

[9] AllSeen-Alliance, https://allseenalliance.org/

[10] J. S. Kwak, J. H. Jin and M. J. Lee, "A Mobile Application for Information Sharing and Collaboration among Co-located People", Proceedings of the 7th International Interdisciplinary Workshop Series, Jeju Island, Korea, August 19-22, (2015).

[11] H. Pennington, A. Carlsson and A. Larsson, "D-Bus Specification Version 0.12.", http://dbus.freedesktop.org/doc/dbus-specification.html, (2007).

\section{Authors}

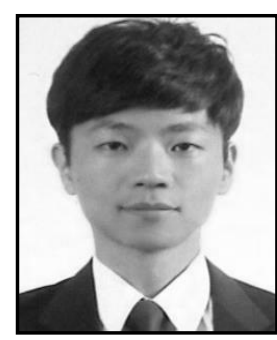

Jun-Seok Kwa, is an M.S. student in the School of Electrical Engineering at the University of Ulsan in Korea. He received his B.S. from the University of Ulsan in Korea. His areas of interest include Internet of Things, mobile service, and network applications.

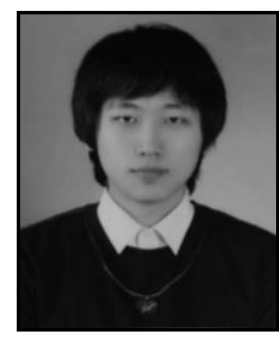

Jae-Hwan Jin, is an M.S. student in the School of Electrical Engineering at the University of Ulsan in Korea. He received his B.S. from the University of Ulsan in Korea. His research interests include Cloud Storage Service, Collaborative System, and Messaging System.

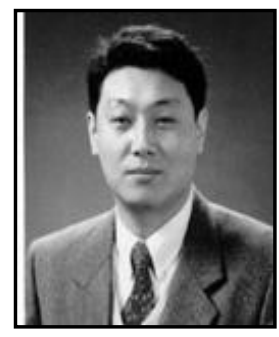

Myung-Joon Lee, is a professor in the School of Electrical Engineering at the University of Ulsan in Korea. He received his $\mathrm{Ph} . \mathrm{D}$. from the Kaist (Korea Advanced Institute of Science and Technology) in Korea. He has (co-)authored more than 200 research publications including numerous works on collaborative system, distributed system and biological system. 
International Journal of Multimedia and Ubiquitous Engineering

Vol.10, No.10 (2015) 